\title{
Spatially Differentiated Trade of Permits for Multipollutant Electric Power Supply Chains
}

\author{
Trisha Woolley ${ }^{1}$, Anna Nagurney ${ }^{2}$, and John Stranlund ${ }^{3}$ \\ 1 Department of Finance and Operations Management, Isenberg School of \\ Management, University of Massachusetts Amherst, Massachusetts, 01003 \\ twoolley@som. umass .edu \\ 2 Department of Finance and Operations Management, Isenberg School of \\ Management, University of Massachusetts Amherst, Massachusetts, 01003 \\ nagurney@gbfin. umass . edu \\ 3 Department of Resource Economics, College of Natural Resources and the \\ Environment University of Massachusetts Amherst, Massachusetts, 01003 \\ stranlund@resecon. umass.edu
}

To appear in Optimization in the Energy Industry, Kallrath J, Pardalos P, Rebennack S, Scheidt M (editors). Berlin, Germany: Springer, in press.

\section{Introduction}

Electric power plants emit several different air pollutants, such as carbon dioxide (CO2), sulfur dioxide (SO2), nitrous oxide ( $\mathrm{NOx})$, and mercury $(\mathrm{Hg})$ with differing environmental impacts . For example, carbon dioxide is a major cause of global climate change; sulfur dioxide and nitrous oxide are responsible for acid rain and fine particle concentrations in the atmosphere; nitrous oxide also contributes to ground-level ozone, and mercury may travel vast distances before deposited in, for example, waterways, bioaccumulating in the food chain resulting in impaired neurological development [7],[2]. Moreover, SO2, NOx, and $\mathrm{Hg}$ have important spatial characteristics; that is, the impacts of these pollutants depend critically on the location of their sources and where their impacts are realized.

Although most environmental regulations attempt to control one pollutant at a time, integrated multipollutant regulations have advantages over the standard piecemeal approach. Multipollutant approaches can account for the substitutability or complementarity of emissions from power plants. As one pollutant is reduced, another may rise, as in, for example, if an electric power generating firm invests in low sulfur coal to reduce SO2 emissions, this will result in an increased amount of NOx and $\mathrm{Hg}$ emissions [31], [32]. How- 
ever, to exploit the complementarity effects of pollutants, firms may invest in electrostatic precipitators (EPSs) that will reduce $\mathrm{SO} 2$ and NOx together. Thus, a generator will choose a technology that is not the cheapest, but reduces multiple pollutants while meeting the current pollutant standard [33]. Furthermore, the relationship between pollutants may vary between seasons, across regions, and, possibly, over time as the composition of the atmosphere changes [36].

Because of such advantages, there have been several existing and proposed regulations to control multiple pollutants. The Regional Clean Air Incentives Market (RECLAIM) program was implemented in California to control NOx and SO2 pollutants; the proposed but not enacted Clear Skies was a national cap to reduce $\mathrm{SO} 2, \mathrm{NOx}$, and $\mathrm{Hg}$; and the US Environmental Protection Agency's Clean Air Interstate Rule (CAIR) capped emissions of SO2 and NOx in a large region covering more than 20 states, mostly east of the Mississippi, and the District of Columbia [30].

Pollution by electric power entities can be controlled by price, in the form, for example, of a carbon tax that is imposed for emissions that exceed a predetermined bound, or by quantity, as in the case of emission trading schemes (cf. [5], [37], [23], and the references therein). There are two types of emission trading policies, project-based (generators purchase credits from a project aimed to reduce emissions) and an allowance market (also known as cap and trade programs). In the latter type, electric power generators are given credits (or allowances) by a central environmental authority. The advantage of emissions trading is that credit trading generates pollution prices that distribute emissions control in a cost-effective manner. For additional background on tradable pollution permits, see [34], [35], [21], [26], [14], [15], and [5].

In this paper, we model the trading of emission rights by electric power producers who emit multiple pollutants with impacts that depend on the spatial dispersion of sources and receptors (for additional background on the electric power industry and associated modeling issues, see [8], [12], [38], [11], [1], [25], and [24]. The control of multiple, spatially differentiated pollutants via emission trading calls for multiple pollution permit markets. Moreover, unlike the previous literature, we emphasize the use of alternative power production technologies as well as the underlying supply chain aspects of electric power generation and distribution. The results in this paper are particularly relevant given the current trends in environmental policies governing emissions in the electric power industry. The new model allows for the determination of the equilibrium numbers and prices of the various tradable pollution permits simultaneously with the equilibrium electric power flows and prices. The model builds upon the electric power supply chain model with alternative power plant technologies developed by $\mathrm{Wu}$ et al. (2006), which, however, only considered a single pollutant (and, in effect, a single receptor point). The model developed by [37] was further transformed into a transportation network equilibrium model (see also, e.g. [22]). 
This paper is organized as follows. In Section 2, we present the model of the electric supply chain network with different power plant technologies and with the inclusion of multipollutant tradable permits with multiple receptor points. We also discuss a special case of the model and demonstrate that the environmental standards are achieved. In Section 3, we describe the computational procedure which exploits the structure of the problem. We also present examples. Section 4 summarizes the results in this paper and presents our conclusions.

\section{The Electric Power Supply Chain Network Model with Multipollutant Tradable Permits}

We now develop the model that captures the behavior of the electric power supply chain network decision-makers in the presence of a multipollutant permit trading scheme. The decision-makers in the electric power supply chain are the electric power generators, with their associated power plants, the suppliers, the transmission service providers, and the consumers at the demand markets. The equilibrium conditions of the electric power supply chain network will be given as well as the equivalent variational inequality formulation.

The electric power supply chain network is represented in Figure 1 with the top tier of nodes consisting of the $G$ power generators (also referred to as "gencos"), enumerated by $1, \ldots, g, \ldots, G$. Power generators are the decisionmakers who own and operate the $M$ power plants, with a typical power plant technology denoted by $m$, and depicted in the second tier of nodes in Figure 1. Such nodes are enumerated as $11, \ldots, G M$ with node $g m$ denoting the $m$-th power plant of genco $g$. The gencos produce electric power using the different power plants, which are powered, for example, by different forms of technology such as coal, natural gas, uranium, oil, sun, wind, etc., and with different associated costs and environmental impacts. The gencos sell the electric power to the power suppliers in the third tier of nodes in the electric power supply chain, as depicted in Figure 1.

In Figure 1, we also represent the $R$ receptor points, with a typical receptor point denoted by $r$, associated with the pollutants generated by the power plants. These receptor points are spatially separated. We also assume that there are $J$ pollutants with a typical pollutant denoted by $j$.

The suppliers do not physically handle the electricity, but function as intermediaries who only hold and trade the right for the electric power. The nodes corresponding to the power suppliers are enumerated as: $1, \ldots, s, \ldots, S$ with node $s$ corresponding to supplier $s$. Suppliers sell the electric power to the consumers at the different demand markets via the $V$ transmission service providers, who are the entities who own and operate the electric power transmission and distribution systems. We denote a typical transmission provider by $v$. 
Transmission service providers are not represented as nodes in the network model, since they do not make decisions such as to where or from whom the electric power will be delivered (see also [24] and [37]). The bottom-tiered nodes in Figure 1 represent the demand markets, which can differ by their geographic location or the type of associated consumers; for example, whether they correspond to businesses or households. The nodes corresponding to the demand markets are enumerated as: $1, \ldots, k, \ldots, K$ with node $k$ corresponding to demand market $k$. The majority of the notation needed for the model is given in Table 1. An equilibrium solution is denoted by "**". All vectors are assumed to be column vectors, except where noted otherwise.

Power Generators

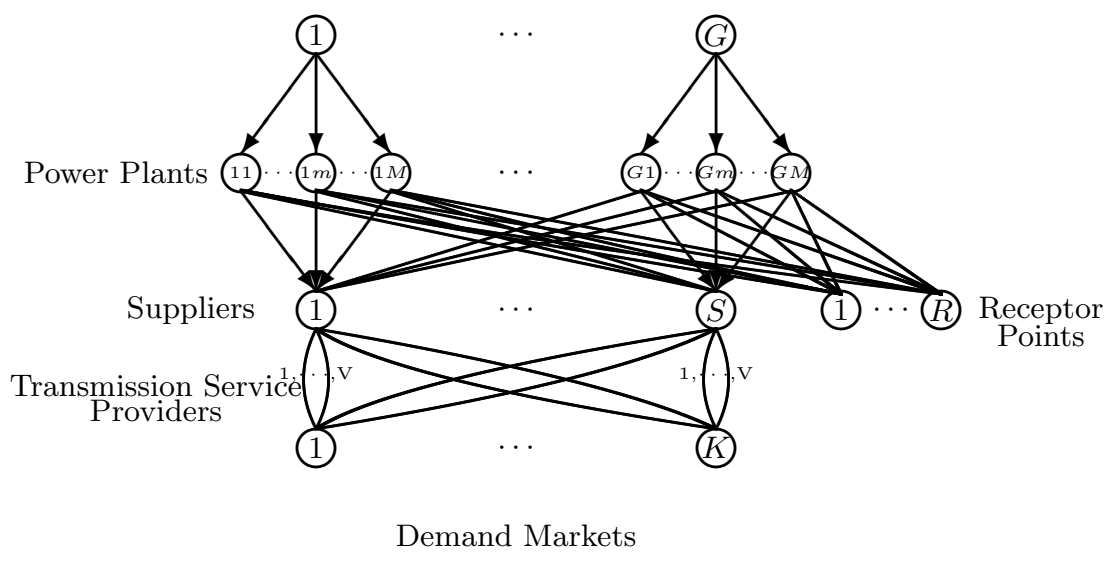

Fig. 1. The Electric Power Supply Chain Network with Power Plants and Associated Technologies and with Pollutant Receptor Points

We now focus on the notation for the permits. Similar to the discussion in [19], [20] and [16], let $l_{g m r}^{j} ; j=1, \ldots, J ; g=1, \ldots, G ; m=1, \ldots, M$; $r=1, \ldots, R$ denote the number of permits/licenses for pollutant of type $j$ held by genco $g$ that uses power plant $m$, and which affects receptor point $r$ with $l_{g m r}^{j 0}$ denoting the initial allocation. Group the former permits into the $J G M R$-dimensional vector $l$.

Let $e_{g m r}^{j} ; j=1, \ldots, J ; g=1, \ldots, G ; m=1, \ldots, M ; r=1, \ldots, R$ denote the unit contribution of the ambient concentration of pollutant type $j$ affecting the receptor point $r$ generated per unit of electric power produced by genco $g$ using his power plant $m$. Hence, the total amount of ambient concentration of pollutant $j$ at receptor point $r$ associated with genco $g$ and power plant $m$ is $e_{g m r}^{j} q_{g m}$. 
Table 1. Notation for the Electric Power Supply Chain Network Model with Power Plants (cf. [37])

\begin{tabular}{|c|c|}
\hline Notation & Definition \\
\hline$q_{g m}$ & $\begin{array}{l}\text { quantity of electricity produced by generator } g \text { using power plant } m \text {, where } \\
g=1, \ldots, G ; m=1, \ldots, M\end{array}$ \\
\hline$q_{m}$ & $\begin{array}{l}G \text {-dimensional vector of electric power generated by the gencos using } \\
\text { power plant technology } m \text { with components: } q_{1 m}, \ldots, q_{G m}\end{array}$ \\
\hline$q$ & $\begin{array}{l}G M \text {-dimensional vector of all the electric power outputs generated } \\
\text { by the gencos at the power plants }\end{array}$ \\
\hline$Q^{1}$ & $\begin{array}{l}G M S \text {-dimensional vector of electric power flows between the power plants of } \\
\text { the power generators and the power suppliers with component gms denoted } \\
\text { by } q_{g m s}\end{array}$ \\
\hline$Q^{2}$ & $\begin{array}{l}S V K \text {-dimensional vector of power flows between suppliers and demand } \\
\text { markets with component } s v k \text { denoted by } q_{s k}^{v} \text { and denoting the flow between } \\
\text { supplier } s \text { and demand market } k \text { via transmission provider } v\end{array}$ \\
\hline$d$ & $K$-dimensional vector of market demands with component $k$ denoted by $d_{k}$ \\
\hline$f_{g m}\left(q_{m}\right)$ & $\begin{array}{l}\text { power generating cost function of power generator } g \text { using power plant } m \\
\text { with marginal power generating cost with respect to } q_{g m} \text { denoted by } \frac{\partial f_{g m}}{\partial q_{g m}}\end{array}$ \\
\hline$c_{g m s}\left(q_{g m s}\right)$ & $\begin{array}{l}\text { transaction cost incurred by power generator } g \text { using power plant } m \\
\text { in transacting with power supplier } s \text { with marginal transaction cost } \\
\text { denoted by } \frac{\partial c_{g m s}\left(q_{g m s}\right)}{\partial q_{g m s}}\end{array}$ \\
\hline$h$ & $\begin{array}{l}S \text {-dimensional vector of the power suppliers' supplies of the electric } \\
\text { power with component } s \text { denoted by } h_{s}, \text { with } h_{s} \equiv \sum_{g=1}^{G} \sum_{m=1}^{M} q_{g m s}\end{array}$ \\
\hline$c_{s}(h) \equiv c_{s}\left(Q^{1}\right)$ & $\begin{array}{l}\text { operating cost of power supplier } s \text { with marginal operating cost with } \\
\text { respect to } h_{s} \text { denoted by } \frac{\partial c_{s}}{\partial h_{s}} \text { and the marginal operating cost with respect } \\
\text { to } q_{g m s} \text { denoted by } \frac{\partial c_{s}\left(Q^{1}\right)}{\partial q_{g m s}}\end{array}$ \\
\hline$c_{s k}^{v}\left(q_{s k}^{v}\right)$ & $\begin{array}{l}\text { transaction cost incurred by power supplier } s \text { in transacting with } \\
\text { demand market } k \text { via transmission provider } v \text { with marginal transaction } \\
\text { cost with respect to } q_{s k}^{v} \text { denoted by } \frac{\partial c_{s k}^{v}\left(q_{s k}^{v}\right)}{\partial q_{s k}^{v}}\end{array}$ \\
\hline$\hat{c}_{g m s}\left(q_{g m s}\right)$ & $\begin{array}{l}\text { transaction cost incurred by power supplier } s \text { in transacting with } \\
\text { power generator } g \text { for power generated by plant } m \text { with marginal transaction } \\
\text { cost denoted by } \frac{\partial \hat{c}_{g m s}\left(q_{g m s}\right)}{\partial q_{g m s}}\end{array}$ \\
\hline$\hat{c}_{s k}^{v}\left(Q^{2}\right)$ & $\begin{array}{l}\text { unit transaction cost incurred by consumers at demand market } k \\
\text { in transacting with power supplier } s \text { via transmission provider } v\end{array}$ \\
\hline$\rho_{3 k}(d)$ & demand market price function at demand market $k$ \\
\hline
\end{tabular}

\subsection{The Behavior of the Power Generators and their Optimality Conditions}

Let $\rho_{1 g m s}^{*}$ denote the unit price charged by power generator $g$ for the transaction with power supplier $s$ for electric power produced at plant $m$ with $g=1, \ldots, G ; m=1, \ldots, M$, and $s=1, \ldots, S . \rho_{1 g m s}^{*}$ is an endogenous variable and can be determined once the complete electric power supply chain 
network equilibrium model is solved. Let $\tau_{r}^{j *} ; j=1, \ldots, J ; r=1, \ldots, R$ denote the price of the permit at equilibrium for pollutant of type $j$ of emission affecting receptor point $r$. These prices are also endogenous to the model and will be determined once the complete model is solved.

We assume that each electric power generator seeks to determine his optimal production portfolio across his power plants and his sales allocations of the electric power to the suppliers as well as the optimal holdings of pollution permits in order to maximize his own profit. Since we have assumed that each individual power generator is a profit-maximizer, the objective function of power generator $g$ can be expressed as follows:

$$
\begin{aligned}
& \text { Maximize } \sum_{m=1}^{M} \sum_{s=1}^{S} \rho_{1 g m s}^{*} q_{g m s}-\sum_{m=1}^{M} f_{g m}\left(q_{m}\right)-\sum_{m=1}^{M} \sum_{s=1}^{S} c_{g m s}\left(q_{g m s}\right) \\
& -\sum_{j=1}^{J} \sum_{m=1}^{M} \sum_{r=1}^{R} \tau_{r}^{j *}\left(l_{g m r}^{j}-l_{g m r}^{j 0}\right) .
\end{aligned}
$$

The first term in the objective function (1) represents the revenue of power generator $g$ and the next two terms represent his power generation cost and transaction costs, respectively. The last term denotes the expenditure or revenue from transacting permits for the generator based on the total pollutants by his power plants affecting the ambient concentrations at the receptor points.

The structure of the network in Figure 1 guarantees that the conservation of flow equations associated with the electric power production and distribution are satisfied. Conservation of flow equation (2) below states that the amount of power generated at a particular power plant (and corresponding to a particular genco) is equal to the electric power transacted by the genco from that power plant with all the suppliers and this holds for each of the power plants, subject to:

$$
\sum_{s=1}^{S} q_{g m s}=q_{g m}, \quad m=1, \ldots, M .
$$

Equation (3) below states that each power plant cannot pollute at an amount greater than the plant is licensed to at that receptor point.

$$
l_{g m r}^{j} \geq e_{g m r}^{j} q_{g m}, \quad j=1 \ldots J ; m=1 \ldots, M ; r=1, \ldots, R .
$$

The following non-negativity conditions must also hold:

$$
\begin{gathered}
q_{g m s} \geq 0, \quad m=1, \ldots, M ; s=1, \ldots, S, \\
l_{g m r}^{j} \geq 0, \quad j=1, \ldots, J ; m=1, \ldots, M ; r=1, \ldots, R .
\end{gathered}
$$


Hence, the optimization problem of power generator $g ; g=1, \ldots, G$ consists of (1), subject to constraints: (2) and (3), with the nonnegativity assumption on the electric power outputs at the power plants and the number of permits (cf. following (1)). Assume now, as was done in [23] and [37], that the generating cost and the transaction cost functions for each power generator are continuously differentiable and convex, and that the power generators compete in a noncooperative manner in the sense of Nash ([28],[29]). The optimality conditions for all power generators, under the above assumptions (cf. [17]), coincide with the solution of the following variational inequality: determine $\left(q^{*}, Q^{1 *}, l^{*}, \lambda^{*}\right) \in \mathcal{K}^{1}$ satisfying

$$
\begin{gathered}
\sum_{g=1}^{G} \sum_{m=1}^{M}\left[\frac{\partial f_{g m}\left(q_{m}^{*}\right)}{\partial q_{g m}}+\sum_{j=1}^{J} \sum_{r=1}^{R} \lambda_{g m r}^{j *} e_{g m r}^{j}\right] \times\left[q_{g m}-q_{g m}^{*}\right] \\
+\sum_{g=1}^{G} \sum_{m=1}^{M} \sum_{s=1}^{S}\left[\frac{\partial c_{g m s}\left(q_{g m s}^{*}\right)}{\partial q_{g m s}}-\rho_{1 g m s}^{*}\right] \times\left[q_{g m s}-q_{g m s}^{*}\right] \\
+\sum_{j=1}^{J} \sum_{g=1}^{G} \sum_{m=1}^{M} \sum_{r=1}^{R}\left[\tau_{r}^{j *}-\lambda_{g m r}^{j *}\right] \times\left[l_{g m r}^{j}-l_{g m r}^{j *}\right] \\
+\sum_{j=1}^{J} \sum_{g=1}^{G} \sum_{m=1}^{M} \sum_{r=1}^{R}\left[l_{g m r}^{j *}-e_{g m r}^{j} q_{g m}^{*}\right] \times\left[\lambda_{g m r}^{j}-\lambda_{g m r}^{j *}\right] \geq 0, \quad \forall\left(q, Q^{1}, l, \lambda\right) \in \mathcal{K}^{1},
\end{gathered}
$$

where $\mathcal{K}^{1} \equiv\left\{\left(q, Q^{1}, l, \lambda\right) \mid\left(q, Q^{1}, l, \lambda\right) \in R_{+}^{G M+G M S+2 J G M R}\right.$ and (2) holds $\}$.

Note that $\lambda_{g m r}^{j}$ is the Lagrange multiplier associated with the $(j m r)$-th constraint (3), which we refer to as a shadow price.

\section{Equilibrium Conditions for the Permits}

Furthermore, we know that (cf. [5]) the multipollutant permit market is also subject to equilibrium conditions given by the following. For each pollution permit of type $j ; j=1, \ldots, J$ and receptor point $r ; r=1, \ldots, R$, a multipollutant tradable permit scheme is said to be in equilibrium if:

$$
\sum_{g=1}^{G} \sum_{m=1}^{M}\left[l_{g m r}^{j 0}-l_{g m r}^{j *}\right] \begin{cases}=0, \text { if } & \tau_{r}^{j *}>0, \\ \geq 0, \text { if } & \tau_{r}^{j *}=0 .\end{cases}
$$

Expression (6) states that if the market price of a permit for pollutant of type $j$ and receptor point $r$ is positive, then there is no excess of permits for that pollutant at that receptor point; if the price is zero, then there can be an excess of such permits. Clearly, these equilibrium conditions guarantee that the total number of required permits cannot exceed the initial allocation of permits by the regulatory agency for each receptor point and pollutant. 
The optimality conditions for all power generators simultaneously (cf. (5)), under the above assumptions (cf. [17]), coupled with the equilibrium conditions ( $(6)$ for all pollutant types and receptor points, coincide, in turn, with the solution of the following variational inequality: determine $\left(q^{*}, Q^{1 *}, l^{*}, \lambda^{*}, \tau^{*}\right) \in$ $\mathcal{K}^{2}$ satisfying

$$
\begin{gathered}
\sum_{g=1}^{G} \sum_{m=1}^{M}\left[\frac{\partial f_{g m}\left(q_{m}^{*}\right)}{\partial q_{g m}}+\sum_{j=1}^{J} \sum_{r=1}^{R} \lambda_{g m r}^{j *} e_{g m r}^{j}\right] \times\left[q_{g m}-q_{g m}^{*}\right] \\
+\sum_{g=1}^{G} \sum_{m=1}^{M} \sum_{s=1}^{S}\left[\frac{\partial c_{g m s}\left(q_{g m s}^{*}\right)}{\partial q_{g m s}}-\rho_{1 g m s}^{*}\right] \times\left[q_{g m s}-q_{g m s}^{*}\right] \\
+\sum_{j=1}^{J} \sum_{g=1}^{G} \sum_{m=1}^{M} \sum_{r=1}^{R}\left[\tau_{r}^{j *}-\lambda_{g m r}^{j *}\right] \times\left[l_{g m r}^{j}-l_{g m r}^{j *}\right] \\
+\sum_{j=1}^{J} \sum_{g=1}^{G} \sum_{m=1}^{M} \sum_{r=1}^{R}\left[l_{g m r}^{j *}-e_{g m r}^{j} q_{g m}^{*}\right] \times\left[\lambda_{g m r}^{j}-\lambda_{g m r}^{j *}\right] \\
+\sum_{j=1}^{J} \sum_{r=1}^{R}\left[\sum_{g=1}^{G} \sum_{m=1}^{M}\left(l_{g m r}^{j 0}-l_{g m r}^{j *}\right)\right] \times\left[\tau_{r}^{j}-\tau_{r}^{j *}\right] \geq 0, \quad \forall\left(q, Q^{1}, l, \lambda, \tau\right) \in \mathcal{K}^{2},
\end{gathered}
$$

where $\mathcal{K}^{2} \equiv\left\{\left(q, Q^{1}, l, \lambda, \tau\right) \mid\left(q, Q^{1}, l, \lambda, \tau\right) \in R_{+}^{G M+G M S+2 J G M R+J R}\right.$

and (2) holds\}.

\section{The Behavior of Power Suppliers and their Optimality Conditions}

The power suppliers transact with the power generators and with the consumers at the demand markets through the transmission service providers. Suppliers are aware as to the types of power plants used and associated costs when purchasing electric power from the power generators. Analogous to the gencos, we assume that the power suppliers compete with one another in a noncooperative manner.

Since electric power cannot be stored, the following conservation of flow constraint states that the total amount of electricity sold by a power supplier is equal to the total electric power that he purchased from the generators and produced via the different power plants available to the generators, that is:

$$
\sum_{k=1}^{K} \sum_{v=1}^{V} q_{s k}^{v}=\sum_{g=1}^{G} \sum_{m=1}^{M} q_{g m s}, \quad s=1, \ldots, S .
$$

Let $\rho_{2 s k}^{v *}$ denote the price charged by power supplier $s$ to demand market $k$ via transmission service provider $v$. This price is determined endogenously in the model once the entire network equilibrium problem is solved. It is 
assumed that each power supplier seeks to maximize his own profit, hence the optimization problem faced by supplier $s$ may be expressed as follows:

$$
\begin{gathered}
\text { Maximize } \sum_{k=1}^{K} \sum_{v=1}^{V} \rho_{2 s k}^{v *} q_{s k}^{v}-c_{s}\left(Q^{1}\right)-\sum_{g=1}^{G} \sum_{m=1}^{M} \rho_{1 g m s}^{*} q_{g m s}-\sum_{g=1}^{G} \sum_{m=1}^{M} \hat{c}_{g m s}\left(q_{g m s}\right) \\
-\sum_{k=1}^{K} \sum_{v=1}^{V} c_{s k}^{v}\left(q_{s k}^{v}\right)
\end{gathered}
$$

subject to:

$$
\begin{gathered}
\sum_{k=1}^{K} \sum_{v=1}^{V} q_{s k}^{v}=\sum_{g=1}^{G} \sum_{m=1}^{M} q_{g m s}, \\
q_{g m s} \geq 0, \quad g=1, \ldots, G ; m=1, \ldots, M, \\
q_{s k}^{v} \geq 0 ; \quad k=1, \ldots, K ; v=1, \ldots, V .
\end{gathered}
$$

The first term in (9) denotes the revenue of supplier $s$ from the sale of electricity to the demand market $k$ via transmission service provider $v$, with the associated operating cost in the second term. The third term denotes the cost to purchase electricity for each supplier from each genco, and the last two terms represent the associated transaction costs for transactions with each genco and each demand market, respectively.

We assume that the transaction costs and the operating costs in (9) are all continuously differentiable and convex, and that the power suppliers compete in a noncooperative manner. Hence, the optimality conditions for all suppliers, simultaneously, under the above assumptions, can be expressed as the following variational inequality: determine $\left(Q^{2 *}, Q^{1 *}\right) \in \mathcal{K}^{3}$ such that

$$
\begin{gathered}
\sum_{s=1}^{S} \sum_{k=1}^{K} \sum_{v=1}^{V}\left[\frac{\partial c_{s k}^{v}\left(q_{s k}^{v *}\right)}{\partial q_{s k}^{v}}-\rho_{2 s k}^{v *}\right] \times\left[q_{s k}^{v}-q_{s k}^{v *}\right] \\
+\sum_{g=1}^{G} \sum_{m=1}^{M} \sum_{s=1}^{S}\left[\frac{\partial c_{s}\left(Q^{1 *}\right)}{\partial q_{g m s}}+\frac{\partial \hat{c}_{g m s}\left(q_{g m s}^{*}\right)}{\partial q_{g m s}}+\rho_{1 g m s}^{*}\right] \times\left[q_{g m s}-q_{g m s}^{*}\right] \geq 0, \text { (12) } \\
\forall\left(Q^{2}, Q^{1}\right) \in \mathcal{K}^{3}, \text { where } \mathcal{K}^{3} \equiv\left\{\left(Q^{2}, Q^{1}\right) \mid\left(Q^{2}, Q^{1}\right) \in R_{+}^{S V K+G M S}\right. \text { and (10); } \\
\text { equivalently (10) holds }\} .
\end{gathered}
$$

For notational convenience, and as was done in [37], we let

$$
h_{s} \equiv \sum_{g=1}^{G} \sum_{m=1}^{M} q_{g m s}, \quad s=1, \ldots, S .
$$

As defined in Table 1, the operating cost of power supplier $s, c_{s}$, is a function of the total electricity inflows to the power supplier, that is: 


$$
c_{s}(h) \equiv c_{s}\left(Q^{1}\right), \quad s=1, \ldots, S .
$$

Hence, his marginal cost with respect to $h_{s}$ is equal to the marginal cost with respect to $q_{g m s}$ :

$$
\frac{\partial c_{s}(h)}{\partial h_{s}} \equiv \frac{\partial c_{s}\left(Q^{1}\right)}{\partial q_{g m s}}, \quad s=1, \ldots, S ; m=1, \ldots, M ; g=1, \ldots, G .
$$

After the substitution of (13) and (15) into (12), and algebraic simplification, we obtain a variational inequality equivalent to (12), as follows: determine $\left(h^{*}, Q^{2 *}, Q^{1 *}\right) \in \mathcal{K}^{4}$ such that

$$
\begin{aligned}
& \sum_{s=1}^{S} \frac{\partial c_{s}\left(h^{*}\right)}{\partial h_{s}} \times\left[h_{s}-h_{s}^{*}\right]+\sum_{s=1}^{S} \sum_{k=1}^{K} \sum_{v=1}^{V}\left[\frac{\partial c_{s k}^{v}\left(q_{s k}^{v *}\right)}{\partial q_{s k}^{v}}-\rho_{2 s k}^{v *}\right] \times\left[q_{s k}^{v}-q_{s k}^{v *}\right] \\
& +\sum_{g=1}^{G} \sum_{m=1}^{M} \sum_{s=1}^{S}\left[\frac{\partial \hat{c}_{g m s}\left(q_{g m s}^{*}\right)}{\partial q_{g m s}}+\rho_{1 g m s}^{*}\right] \times\left[q_{g m s}-q_{g m s}^{*}\right] \geq 0, \\
& \forall\left(h, Q^{2}, Q^{1},\right) \in \mathcal{K}^{4}, \text { where } \mathcal{K}^{4} \equiv\left\{\left(h, Q^{2}, Q^{1}\right) \mid\left(h, Q^{2}, Q^{1}\right) \in R_{+}^{S(1+V K+G M)}\right. \\
& \text { and }(10) \text { and }(13) \text { hold }\} .
\end{aligned}
$$

\section{Equilibrium Conditions for the Demand Markets}

At each demand market $k$ the following conservation of flow equation must be satisfied:

$$
d_{k}=\sum_{s=1}^{S} \sum_{v=1}^{V} q_{s k}^{v}, \quad k=1, \ldots, K .
$$

For each power supplier $s ; s=1, \ldots, S$ and transaction mode $v ; v=1, \ldots, V$, the market equilibrium conditions at demand market $k$ take the form:

$$
\rho_{2 s k}^{v *}+\hat{c}_{s k}^{v}\left(Q^{2 *}\right) \begin{cases}=\rho_{3 k}\left(d^{*}\right), \text { if } & q_{s k}^{v *}>0, \\ \geq \rho_{3 k}\left(d^{*}\right), \text { if } & q_{s k}^{v *}=0 .\end{cases}
$$

According to [24], [23], and [37], consumers at the demand market will purchase electricity from a supplier via a transmission service provider if the price that the consumer at the demand market is willing to pay is equal to the price charged by the power supplier plus the unit transaction cost. However, if the purchase price plus the unit transaction cost exceeds the purchase price that the consumer is willing to pay, then no transaction will take place. The equivalent variational inequality, given that, in equilibrium, condition (18) must hold simultaneously for all demand markets: $k=1, \ldots, K$, takes the form: determine $\left(Q^{2 *}, d^{*}\right) \in \mathcal{K}^{5}$, such that

$$
\begin{gathered}
\sum_{s=1}^{S} \sum_{k=1}^{K} \sum_{v=1}^{V}\left[\rho_{2 s k}^{v *}+\hat{c}_{s k}^{v}\left(Q^{2 *}\right)\right] \times\left[q_{s k}^{v}-q_{s k}^{v *}\right]-\sum_{k=1}^{K} \rho_{3 k}\left(d^{*}\right) \times\left[d_{k}-d_{k}^{*}\right] \geq 0, \\
\forall\left(Q^{2}, d\right) \in \mathcal{K}^{5}, \text { where } \mathcal{K}^{5} \equiv\left\{\left(Q^{2}, d\right) \mid\left(Q^{2}, d\right) \in R_{+}^{K S V+K} \text { and (17) holds }\right\} .
\end{gathered}
$$


The Equilibrium Conditions for the Electric Power Supply Chain Network with Multipollutant Permits

In equilibrium, the optimality conditions for all the power generators, the optimality conditions for all the power suppliers, and the equilibrium conditions for all the demand markets as well as the equilibrium conditions for the permits must be simultaneously satisfied so that no decision-maker has any incentive to alter his transactions. We now formally state the equilibrium conditions for the entire electric power supply chain network along with the variational inequality formulation, which follows directly from the definition.

\section{Definition 1: Electric Power Supply Chain Network Equilibrium with Multipollutant Permits}

The equilibrium state of the electric power supply chain network with power plants and multipollutant permits is one where the electric power flows between the tiers of the network coincide and the electric power flows and the multipollutant tradable permits and prices satisfy the sum of conditions (5), (19), and (19).

\section{Variational Inequality Formulation of the Electric Power Supply Chain Network Equilibrium with Multipollutant Permits}

The equilibrium conditions governing the electric power supply chain network according to Definition 1 coincide with the solution of the variational inequality given by: determine the vector of equilibrium electric power production quantities and flows, the demands, the number of permits, the shadow prices, and the permit prices $\left(q^{*}, h^{*}, Q^{1 *}, Q^{2 *}, d^{*}, l^{*}, \lambda^{*}, \tau^{*}\right) \in \mathcal{K}^{6}$ satisfying:

$$
\begin{gathered}
\sum_{g=1}^{G} \sum_{m=1}^{M}\left[\frac{\partial f_{g m}\left(q_{m}^{*}\right)}{\partial q_{g m}}+\sum_{j=1}^{J} \sum_{r=1}^{R} \lambda_{g m r}^{j *} e_{g m r}^{j}\right] \times\left[q_{g m}-q_{g m}^{*}\right]+\sum_{s=1}^{S} \frac{\partial c_{s}\left(h^{*}\right)}{\partial h_{s}} \times\left[h_{s}-h_{s}^{*}\right] \\
+\sum_{g=1}^{G} \sum_{m=1}^{M} \sum_{s=1}^{S}\left[\frac{\partial c_{g m s}\left(q_{g m s}^{*}\right)}{\partial q_{g m s}}+\frac{\partial \hat{c}_{g m s}\left(q_{g m s}^{*}\right)}{\partial q_{g m s}}\right] \times\left[q_{g m s}-q_{g m s}^{*}\right] \\
+\sum_{s=1}^{S} \sum_{k=1}^{K} \sum_{v=1}^{V}\left[\frac{\partial c_{s k}^{v}\left(q_{s k}^{v *}\right)}{\partial q_{s k}^{v}}+\hat{c}_{s k}^{v}\left(Q^{2 *}\right)\right] \times\left[q_{s k}^{v}-q_{s k}^{v *}\right]-\sum_{k=1}^{K} \rho_{3 k}\left(d^{*}\right) \times\left[d_{k}-d_{k}^{*}\right] \\
+\sum_{j=1}^{J} \sum_{g=1}^{G} \sum_{m=1}^{M} \sum_{r=1}^{R}\left[\tau_{r}^{j *}-\lambda_{g m r}^{j *}\right] \times\left[l_{g m r}^{j}-l_{g m r}^{j *}\right] \\
+\sum_{j=1}^{J} \sum_{g=1}^{G} \sum_{m=1}^{M} \sum_{r=1}^{R}\left[l_{g m r}^{j *}-e_{g m r}^{j} q_{g m}^{*}\right] \times\left[\lambda_{g m r}^{j}-\lambda_{g m r}^{j *}\right]
\end{gathered}
$$


$+\sum_{j=1}^{J} \sum_{r=1}^{R}\left[\sum_{g=1}^{G} \sum_{m=1}^{M}\left(l_{g m r}^{j 0}-l_{g m r}^{j *}\right)\right] \times\left[\tau_{r}^{j}-\tau_{r}^{j *}\right] \geq 0, \forall\left(q, h, Q^{1}, Q^{2}, d, l, \lambda, \tau\right) \in \mathcal{K}^{6}$,

where $\mathcal{K}^{6} \equiv\left\{\left(q, h, Q^{1}, Q^{2}, d, l, \lambda, \tau\right) \mid\left(q, h, Q^{1}, Q^{2}, d, l, \lambda, \tau\right)\right.$ $\in R_{+}^{G M+S+G M S+S K V+K+2 J G M R+J R}$ and (2), (10), (13), and (17) hold\}.

We now put variational inequality (20) into standard form (cf. [17]), which can be expressed as:

$$
\left\langle F\left(X^{*}\right), X-X^{*}\right\rangle \geq 0, \quad \forall X \in \mathcal{K},
$$

where $X \equiv\left(q, h, Q^{1}, Q^{2}, d, l, \lambda, \tau\right) \in R_{+}^{G M+S+G M S+S K V+K+2 G M R J+R J}$ and $F(X)$ as a column vector consisting of the column vectors $\left(P_{g m}, H_{s}, \Lambda_{g m s}\right.$, $\left.G_{s k v}, D_{k}, L_{j g m r}, C_{j g m r}, T_{j r}\right)$ with indices: $g=1, \ldots, G ; m=1, \ldots, M$; $s=1, \ldots, S ; k=1, \ldots, K ; v=1, \ldots, V ; j=1, \ldots, J ; r=1, \ldots, R$, and the specific components of $\mathrm{F}$ given by the functional terms preceding the multiplication signs in (20), respectively. The term $\langle\cdot, \cdot\rangle$ denotes the inner product in $N$-dimensional Euclidean space $R^{N}$.

We now identify a special case of the above model which will correspond to a particular pollution permit trading scheme. Chen and Hobbs ([3]; also see [4]) considered a single pollutant and single receptor point tradable permit market scheme. We now provide additional theoretical results which are important for environmental decision-making and policy-making. Similar results can be found in [5], but not generalized to the electric power industry with multiple power plants. Let $\bar{E}_{r}^{j} ; j=1, \ldots, J ; r=1, \ldots, R$, denote the imposed environmental standard for receptor $r$ and emission type $j$. We now state the following.

\section{Theorem 1 (Equilibrium Pattern Independence from Initial Permit Allocation)}

If $l_{g m r}^{j 0} \geq 0$, for all $j=1, \ldots, J ; g=1, \ldots, G ; m=1, \ldots, M$, and $r=$ $1, \ldots, R$, and $\sum_{g=1}^{G} \sum_{m=1}^{M} l_{g m r}^{j 0}=\bar{E}_{r}^{j}$, for $j=1, \ldots, J ; r=1, \ldots, R$ with each $\bar{E}_{r}^{j}$ positive and fixed, then the equilibrium pattern $\left(q^{*}, h^{*}, Q^{1 *}, Q^{2 *}, d^{*}, l^{*}, \lambda^{*}, \tau^{*}\right)$ is independent of $\left\{l_{g m r}^{j 0}\right\}$.

\section{Proof:}

The last term in (20) (unlike the first seven in (20) which are independent of $l_{g m r}^{j 0}$ ) depends only on the sum $\sum_{g=1}^{G} \sum_{m=1}^{M} l_{g m r}^{j 0}$, for a fixed receptor point $j$ and a fixed pollutant of type $j$.

In the next Theorem, we provide a means for the selection of the sums of the initial permit/license allocation so that the imposed environmental standards are achieved. 


\section{Theorem 2 (Attainment of Environmental Standards)}

An equilibrium vector, satisfying variational inequality (20), attains the environmental quality standards represented by vector $\bar{E}=\left(\bar{E}_{1}, \ldots, \bar{E}_{R}\right)$ where $\bar{E}_{r}=\left(\bar{E}_{r}^{1}, \ldots, \bar{E}_{r}^{J}\right)$ for $r ; r=1, \ldots, R$, provided that the following is satisfied:

$$
\sum_{g=1}^{G} \sum_{m=1}^{M} l_{g m r}^{j 0}=\bar{E}_{r}^{j}, \quad \forall r, \forall j .
$$

\section{Proof:}

From constraint (3) we have that

$$
l_{g m r}^{j *} \geq e_{g m r}^{j} q_{g m}^{*}, \quad j=1 \ldots J ; m=1 \ldots, M ; r=1, \ldots, R .
$$

it then follows from equilibrium conditions (6) that

$$
\bar{E}_{r}^{j}=\sum_{g=1}^{G} \sum_{m=1}^{M} l_{g m r}^{j 0} \geq \sum_{g=1}^{G} \sum_{m=1}^{M} l_{g m r}^{j *} \geq \sum_{g=1}^{G} \sum_{m=1}^{M} e_{g m r}^{j} q_{g m}^{*}
$$

for all $j=1, \ldots, J ; r=1, \ldots, R$.

Theorem 2 provides a mechanism for the determination of the sums of the initial permit/license allocations so that the environmental standards are attained. Indeed, all one needs to do is to set the initial permit allocation so that (22) is satisfied. We will illustrate this with examples in the next section.

\section{Algorithm and Examples}

Clearly, there are distinct variational inequality algorithms that may be applied to solve variational inequalities (20), and, in particular, we note the modified projection method (see [17]) which was been successfully applied to solve variational inequality problems in which the function $F$ (cf. (21)) is monotone and Lipschitz continuous.

Wu et al. ([37]) in turn, proposed an Euler method for the electric power supply chain network equilibrium problem with power plants and reassigned carbon taxes. That Euler method was introduced by Dupuis and Nagurney ([6]), and is a special case of a general iterative scheme for the solution of variational inequalities as well as projected dynamical systems. [37] showed that the electric power supply chain problem with preassigned taxes could be transformed into a transportation network equilibrium problem over an appropriately constructed abstract network or supernetwork. 
In the model and special case developed in this paper, we can no longer transform the variational inequalities (20) directly into transportation network equilibrium problems as was also done by [18] for supply chain network equilibrium problems. However, we can still exploit the connection by noticing that the variational inequality problems in this paper are defined over feasible sets that are, in effect, decomposable into subproblems in the flows and subproblems in the licenses, the shadow prices, and the license prices. Furthermore, the former subproblems retain the transportation network structure identified in [37] and this can be exploited algorithmically. Hence, we can apply the Euler method, whose general statement to solve a variational inequality given by: determine $X^{*} \in \mathcal{K}$ such that

$$
\left\langle F\left(X^{*}\right), X-X^{*}\right\rangle \geq 0, \quad \forall X \in \mathcal{K},
$$

and is given immediately following.

\section{The Euler Method}

The Euler method (see [6]) has been applied by [27] to solve the variational inequality governing elastic demand transportation network equilibrium problems in path flows. Convergence results can be found in the above references. For the solution of (25), the Euler method takes the form: at iteration $l$ compute $X^{l+1}$ by solving the variational inequality problem:

$$
X^{l+1}=P_{\mathcal{K}}\left(X^{l}-a_{l} F\left(X^{l}\right)\right),
$$

where $P_{\mathcal{K}}$ is the projection operator, and the sequence $\left\{a_{l}\right\}$ must satisfy the conditions: $\sum_{l=0}^{\infty} a_{l}=\infty, a_{l}>0$, for all $l$, and $a_{l} \rightarrow 0$, as $l \rightarrow \infty$.

For completeness, we now present several examples. The examples consisted of two power generators, each of which had two power plants. There were two power suppliers and two demand markets with a single transmission provider. We also assumed that there was a single pollutant and a single receptor point, as shown in Figure 2.

\section{Example 1}

The data for the first example are given below. The functional forms of the power generating cost functions, the transaction cost functions, the operating cost functions, and the demand price functions are identical to those in Example 1 in [37].

The emission terms: $e_{g m} ; g=1,2 ; m=1,2$ were all equal to 1 . The power generating cost functions for the power generators were given by:

$$
\begin{gathered}
f_{11}\left(q_{1}\right)=2.5 q_{11}^{2}+q_{11} q_{21}+2 q_{11}, \quad f_{12}\left(q_{2}\right)=2.5 q_{12}^{2}+q_{11} q_{12}+2 q_{22}, \\
f_{21}\left(q_{1}\right)=.5 q_{21}^{2}+.5 q_{11} q_{21}+2 q_{21}, \quad f_{22}\left(q_{2}\right)=.5 q_{22}^{2}+q_{12} q_{22}+2 q_{22} .
\end{gathered}
$$


The transaction cost functions faced by the power generators and associated with transacting with the power suppliers were given by:

$$
\begin{array}{cl}
c_{111}\left(q_{111}\right)=.5 q_{111}^{2}+3.5 q_{111}, & c_{112}\left(q_{112}\right)=.5 q_{112}^{2}+3.5 q_{112}, \\
c_{121}\left(q_{121}\right)=.5 q_{121}^{2}+3.5 q_{121}, & c_{122}\left(q_{122}\right)=.5 q_{122}^{2}+3.5 q_{122}, \\
c_{211}\left(q_{211}\right)=.5 q_{211}^{2}+2 q_{211}, & c_{212}\left(q_{212}\right)=.5 q_{212}^{2}+2 q_{212}, \\
c_{221}\left(q_{221}\right)=.5 q_{221}^{2}+2 q_{221}, & c_{222}\left(q_{222}\right)=.5 q_{222}^{2}+2 q_{222} .
\end{array}
$$

The operating costs of the power generators, in turn, were given by:

$$
c_{1}\left(Q^{1}\right)=.5\left(\sum_{i=1}^{2} q_{i 1}\right)^{2}, \quad c_{2}\left(Q^{1}\right)=.5\left(\sum_{i=1}^{2} q_{i 2}\right)^{2} .
$$

Power Generators

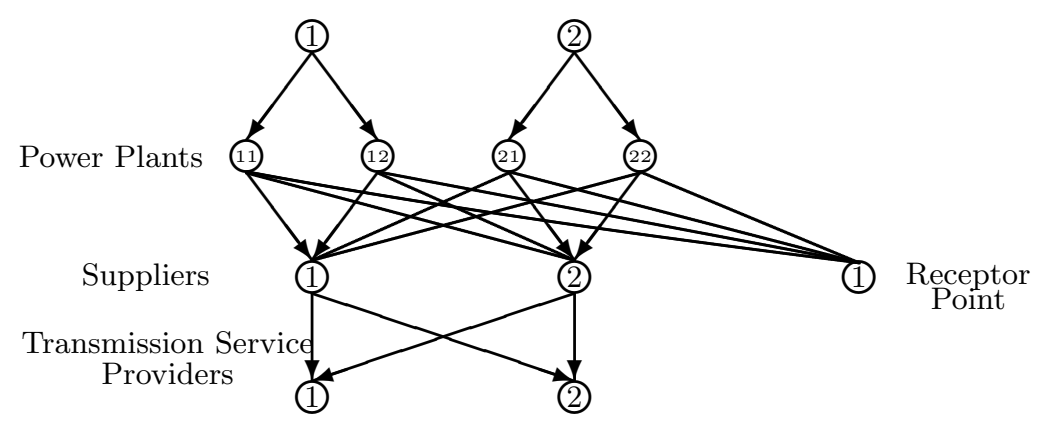

Demand Markets

Fig. 2. Electric Power Supply Chain Network with a Single Receptor Point for the Examples

The demand market price functions at the demand markets were:

$$
\rho_{31}(d)=-1.33 d_{1}+366.6, \quad \rho_{32}=-1.33 d_{2}+366.6,
$$

and the transaction costs between the power suppliers and the consumers at the demand markets were given by: $\hat{c}_{s k}^{1}\left(q_{s k}^{1}\right)=q_{s k}^{1}+5, \quad s=1,2 ; k=1,2$. All other transaction costs were assumed to be equal to zero.

In Example 1, the emissions standard $\bar{E}=100$ with the initial license allocation given by: $l_{11}^{0}=l_{12}^{0}=l_{21}^{0}=l_{22}^{0}=25$. The equilibrium electric power flows and demands and the equilibrium licenses and prices are given in Table 2. The demand was 50.00 at each demand market and the demand market price at each market for electric power was 300.10. 


\section{Example 2}

Example 2 had the same data as Example 1, but we now tightened the emissions standard so that $\bar{E}=50$. The initial license allocation was now given by: $l_{11}^{0}=l_{12}^{0}=l_{21}^{0}=l_{22}^{0}=12.5$. The equilibrium solution is given in Table 2. It is clear that, as predicted by the theory, the environmental standard is achieved.

\section{Example 3}

Example 3 had the identical data to that in Examples 1 and 2, except that the environmental standard was further tightened to $\bar{E}=20$ with the new initial license allocation given by: $l_{11}^{0}=l_{12}^{0}=l_{21}^{0}=l_{22}^{0}=5$. The new equilibrium pattern is reported in Table 2. In this example, it is also clear that the equilibrium license numbers are such that the environmental standard is attained.

\section{Example 4}

Example 4 had the same data as Example 3 except that we modified the second demand market price function for electric power to:

$$
\rho_{32}(d)=-1.33 d_{2}+733.30 .
$$

The new equilibrium electric power flow, license, and price pattern is also reported in Table 2. In this example, there is zero demand for electric power at the first demand market. As in the preceding examples, the environmental standard is achieved. Note that as the equilibrium price of the permits increases, as expected, as the environmental standard is tightened for each successive example. 


\begin{tabular}{|c|c|c|c|c|}
\hline Equilibrium Solution & \multicolumn{4}{|c|}{\begin{tabular}{|l|l|l|l|} 
Example 1 & Example 2 & Example 3 & Example 4 \\
\end{tabular}} \\
\hline \multicolumn{5}{|c|}{ Equilibrium Electric Power Flows } \\
\hline$q_{11}^{*}$ & 15.20 & 7.48 & 2.85 & 2.87 \\
\hline$q_{12}^{*}$ & 6.63 & 3.17 & 1.10 & 1.10 \\
\hline$q_{21}^{*}$ & 15.53 & 7.82 & 3.19 & 3.20 \\
\hline$q_{22}^{*}$ & 62.65 & 31.53 & 12.86 & 12.91 \\
\hline$q_{111}^{*}$ & 7.60 & 3.74 & 1.43 & 1.43 \\
\hline$q_{112}^{*}$ & 7.60 & 3.74 & 1.43 & 1.43 \\
\hline$q_{121}^{*}$ & 3.31 & 1.59 & 0.55 & 0.55 \\
\hline$q_{122}^{*}$ & 3.31 & 1.59 & 0.55 & 0.55 \\
\hline$q_{211}^{*}$ & 7.76 & 3.91 & 1.59 & 1.60 \\
\hline$q_{212}^{*}$ & 7.76 & 3.91 & 1.59 & 1.60 \\
\hline$q_{221}^{*}$ & 31.32 & 15.77 & 6.43 & 6.46 \\
\hline$q_{222}^{*}$ & 31.32 & 15.77 & 6.43 & 6.46 \\
\hline$h_{1}^{*}$ & 50.00 & 25.00 & 10.00 & 10.00 \\
\hline$h_{2}^{*}$ & 50.00 & 25.00 & 10.00 & 10.00 \\
\hline$q_{11}^{1 *}$ & 25.00 & 12.50 & 5.00 & 0.00 \\
\hline$q_{12}^{1 *}$ & 25.00 & 12.50 & 5.00 & 10.00 \\
\hline$q_{21}^{1 *}$ & 25.00 & 12.50 & 5.00 & 0.00 \\
\hline$q_{22}^{1 *}$ & 25.00 & 12.50 & 5.00 & 10.00 \\
\hline \multicolumn{5}{|c|}{ Equilibrium Demands } \\
\hline$d_{1}^{*}$ & 50.00 & 25.00 & 10.00 & 0.00 \\
\hline$d_{2}^{*}$ & 50.00 & 25.00 & 10.00 & 20.00 \\
\hline \multicolumn{5}{|c|}{ Equilibrium Pollution Permit Price and Shadow Prices } \\
\hline$\tau^{*}=\lambda_{11}^{*}=\lambda_{12}^{*}=\lambda_{21}^{*}=\lambda_{22}^{*}$ & 115.50 & 236.38 & 308.91 & 656.96 \\
\hline \multicolumn{5}{|c|}{\begin{tabular}{|l} 
Equilibrium Permits/Licenses \\
\end{tabular}} \\
\hline$l_{11}^{*}$ & 15.20 & 7.48 & 2.85 & 2.87 \\
\hline$l_{12}^{*}$ & 6.63 & 3.17 & 1.10 & 1.10 \\
\hline$l_{21}^{*}$ & 15.53 & 7.82 & 3.19 & 3.20 \\
\hline$l_{22}^{*}$ & 62.65 & 31.53 & 12.86 & 12.91 \\
\hline
\end{tabular}

Table 2. Solutions to Examples 1, 2, 3, and 4

\section{Summary and Conclusions}

As noted in the Introduction, pollution by electric power entities can be controlled by price, in the form, for example, of a carbon tax that is imposed for emissions that exceed a predetermined bound (and as modeled in [37] and [23], or by quantity, as in the case of emission trading schemes. In this paper, we developed a multipollutant permit trading model in the case of electric power supply chains in which there are different technologies associated with electric power production. We derived the governing equilibrium conditions of the model and showed that it satisfies a finite-dimensional variational inequality problem. We also discuss a special case of the model. Moreover, we demonstrated that the model guarantees that the environmental standards are 
achieved, provided that the initial license allocation is set accordingly. Finally, we described how the equilibrium electric power flows and the pollution permits/licenses, along with their prices could be computed. For completeness, we also provided several numerical examples. Future research will include the identification of efficient computational procedures for large-scale electric power supply chains with tradable pollution permits.

The research in this chapter is the first to incorporate the substitutability and complementarity effects of multiple pollutants. This research can aid a regulatory agency in the determination of the number of permits required to achieve the reduction of emissions below a pre-determined bound. Moreover, this model focuses specifically on electric power supply chains and the effects of governmental mandates regarding environmental standards on the associated prices and quantities. The importance of environmental-energy modeling to address market failures in energy is growing as awareness of pollution effects, emission abatement technologies, and government policies are changing. A limitation of the model is the requirement of the electric power industry to report accurate and true data regarding the costs of producing electricity. A future application of this model could include the empirical implementation of a tradable permit system, such as, for example, for the electric power supply chain of New England (see [13]).

\section{Acknowledgments}

The authors are indebted to the two anonymous reviewers for their helpful comments and suggestions on an earlier version of this chapter. The research of the first two authors was supported, in part, by NSF Grant. No.: IIS 00026471

and, in part, by the John F. Smith Memorial Fund at the Isenberg School of Management. This support is gratefully acknowledged and appreciated.

\section{References}

1. Boucher J, Smeers Y (2001) Studies in the Economics of Transportation. Operations Research 49: 821-838

2. Burtraw D, Evans D A, Krupnick A, Palmer K, Toth R (2005) Alternative Models of Restructured Electricity System, Part 1: No Market Power. Annual Review of Environment and Resources 30: 253-289

3. Chen Y H, Hobbs B F (2005) An Oligopolistic Electricity Market model with Tradable NOx Permits. IEEE Transactions on Power Systems 20: 119-129

4. Chen Y H, Hobbs B F, Leyffer S, Munson T S (2006) Leader-follow Equilibria for Electric Power and $\mathrm{NO}_{x}$ Allowances Markets. Computational Management Science Online: 1619-6988

5. Dhanda K K, Nagurney A, Ramanujam P (1999) Environmental Networks: A Framework for Economic Decision-Making and Policy Analysis. Edward Elgar Publishing, Cheltenham England 
6. Dupuis P, Nagurney A (1993) Dynamical Systems and Variational Inequalities. Annals of Operations Research 44: 9-42

7. Hanisch C (1998) Where is Mercury Deposition Coming From? Uncertainties About the Roles of Different Natural and Synthetic Sources are Fueling the Debate on How to Regulate Emissions. Environmental Science and Technology 32: $176 \mathrm{~A}-179 \mathrm{~A}$

8. Hogan W W (1992) Contract Networks for Electric Power Transmission. Journal of Regulatory Economics 4: 211-242

9. Intergovernmental Panel on Climate Change (2001) Synthesis Report: Intergovernmental Panel on Climate Change Third Assessment Report

10. Intergovernmental Panel on Climate Change (2005) Special Report Carbon Dioxide Capture and Storage Summary for Policymakers: A Report of Working Group III of the IPCC and Technical Summary

11. Jing-Yuan W, Smeers Y (1999) Spatial Oligopolistic Electricity Models with Cournot Generators and Regulated Transmission Prices. Operations Research 47: $102-112$

12. Kahn, E P (1998) Numerical Techniques for Analyzing Market Power in Electricity. The Electricity Journal 11: $34-43$

13. Liu Z, Nagurney A (2008) An Integrated Electric Power Supply Chain and Fuel Market Network Framework: Theoretical Modeling with Empirical Analysis for New England. Working Paper, University of Massachusetts Amherst

14. Montero J P (1997) Marketable Pollution Permits with Uncertainty and Transaction Costs. Resource and Energy Economics 20: 27-50

15. Montero J P (2001) Multipollutant Markets. The RAND Journal of Economics 32: $762-774$

16. Montgomery W D (1972) Markets in Licenses and Efficient Pollution Control Programs. Journal of Economic Theory 5: 395-418

17. Nagurney A (1999) Network Economics: A Variational Inequality Approach Second Edition. Kluwer Academic Publishers, Dordrecht, The Netherlands

18. Nagurney A (2006) On the Relationship Between Supply Chain and Transportation Network Equilibria: A Supernetwork Equivalence with Computations. Transportation Research E 42: 293-316

19. Nagurney A, Dhanda K K (1996) Variational Inequality Approach for Marketable Pollution Permits. Computational Economics 9: 363-384

20. Nagurney A, Dhanda K K (2000) Marketable Pollution Permits in Oligopolistic Markets with Transaction Costs. Operations Research 48: 424-435

21. Nagurney A, Dhanda K K, Stranlund J K (1997) A General Multiproduct, Multipollutant Market Pollution Permit Model: A Variational Inequality Approach. Energy Economics 19: 57-76

22. Nagurney A, Liu Z (2005) Transportation Network Equilibrium Reformulations of Electric Power Networks with Computations. Working Paper, University of Massachusetts Amherst

23. Nagurney A, Liu Z, Woolley T (2006) Optimal Endogenous Carbon Taxes for Electric Power Supply Chains with Power Plants. Mathematical and Computer Modelling 44: 899-916

24. Nagurney A, Matsypura D (2005) A Supply Chain Network Perspective for Electric Power Generation, Supply, Transmission, and Consumption. In: Kontoghiorghes E J, Gatu C (eds) Advances in Computational Economics, Finance and Management Science. Springer, Berlin Germany 
25. Nagurney A, Matsypura D (2004) A Supply Chain Network Perspective for Electric Power Generation, Supply, Transmission, and Consumption. Proceedings of the International Conference in Computing, Communications and Control Technologies Volume VI, Austin Texas: 127-134

26. Nagurney A, Ramanujam P, Dhanda K K (1998) A Multimodal Traffic Network Equilibrium Model with Emission Pollution Permits: Compliance versus Noncompliance. Transportation Research D 3: 349-374

27. Nagurney A, Zhang D (1996) Projected Dynamical Systems and Variational Inequalities with Applications. Kluwer Academic Publishers, Boston, Massachusetts

28. Nash J F (1950) Equilibrium Points in N-person Games. Proceedings of the National Academy of Sciences 36: 48-49

29. Nash J F (1951) Noncooperative Games. Annals of Mathematics 54: 286-298

30. Palmer K, Burtraw D, Shih J S (2007) The Benefits and Costs of Reducing Emissions from The Electricity Sector. Journal of Environmental Management 83: $115-130$

31. Rubin, E S, Kalagnanam J R, Frey H C, Barkenpas M B (1997) Integrated Environmental Control Modeling of Coal-fired Power Systems. Journal of Air and Waste Management Association 47: 1180-1188

32. Rubin E S, Berkenpas M B, Farrel A, Gibbon G A, Smith D N (2001) Multipollutant Emission Control of Electric Power Plants. In: Proceedings of EPADOE-EPRI Mega Symposium. Chicago, Illinois.

33. Schwarz P (2005) Multipollutant Efficiency Standards for Electricity Production. Contemporary Economic Policy 23: 341-356

34. Tietenberg T H (1985) Emissions Trading: An Exercise in Reforming Pollution Policy. In: Resources for the Future. Washington DC.

35. Tschirhart J, Wen S (1999) Tradable Allowances in A Restructuring Electric Industry. Journal of Environmental Economics and Management 38: 195-214

36. Western Regional Air Partnership (2005) DRAFT For WRAP Market Trading Forum Review Stationary Source NOx and PM Emissions in the WRAP Region: An Initial Assessment of Emissions, Controls, and Air Quality Impacts.

37. Wu K, Nagurney A, Liu Z, Stranlund J K (2006) Modelling Generator Power Plant Portfolios and Pollution Taxes in Electric Power Supply Chain Networks: A Transportation Network Equilibrium Transformation. Transportation Research D 11: 171-190

38. Zaccour G (2001) Deregulation of Electric Utilities. Deregulation of Electric Utilities. Kluwer Academic Publishers, Boston, Massachusetts. 


\section{Index}

alternative power technologies, 2 ambient concentrations, 6

convex, 9

elastic demand, 14

electric power plants, 1

emission trading policies, 2

environmental impacts, 1

environmental regulations, 1

environmental standards, 13

equilibrium conditions, 7,10

Euler method, 14

modified projection method, 13 multipollutant, 1

Nash equilibrium, 7

optimization, 7

pollutants, 1

receptor points, 6

regulations, 2

supply chain network, 3

tradable permit, 2

variational inequality, 11 